Société d'histoire de la révolution de 1848 et des

révolutions du XIXe siècle

$58 \mid 2019$

Histoire et archéologie : que faire du XIXe siècle ?

\title{
Travailler à l'INRAP : une historienne chez les archéologues
}

Entretien avec Colette Castrucci

Working at the l'INRAP : A historian among archeologue. Interview with Colette Castrucci

Im INRAP arbeiten: eine Historikerin unter Archäologen. Interview mit Colette

Castrucci

Colette Castrucci

\section{CpenEdition}

Journals

Édition électronique

URL : https://journals.openedition.org/rh19/6485

DOI : 10.4000/rh19.6485

ISSN : $1777-5329$

Éditeur

La Société de 1848

Édition imprimée

Date de publication : 1 août 2019

Pagination : 147-158

ISSN : 1265-1354

Référence électronique

Colette Castrucci, «Travailler à l'INRAP : une historienne chez les archéologues », Revue d'histoire du XIXe siècle [En ligne], 58 | 2019, mis en ligne le 01 janvier 2022, consulté le 08 juillet 2022. URL : http:// journals.openedition.org/rh19/6485; DOI : https://doi.org/10.4000/rh19.6485 


\section{Travailler à l'INRAP : une historienne chez les archéologues}

\section{ENTRETIEN AVEC COLETTE CASTRUCCI}

Entretien mené par Manuel Charpy et Stéphanie Sauget avec Colette Castrucci, docteure en histoire moderne, au service de l'Association pour les Fouilles Archéologiques (AFAN), devenue Institut national de la recherche archéologique préventive (INRAP) en 2002, rencontrée en janvier 2017 à l'occasion d'un atelier d'étude sur le thème "Histoire et archéologie : que faire du XIXe siècle?", atelier préparatoire à l'élaboration de ce numéro.

RH19 : Colette Castrucci, comment aimez-vous que l'on vous présente? Comme une historienne ou comme une archéologue? Selon vous la distinction historienne/archéologue fait-elle sens?

Ayant soutenu une thèse en histoire, je suis historienne avant tout ${ }^{1}$. Néanmoins, travaillant pour les archéologues depuis plus de vingt ans, j’ai appris à comprendre ce qu'ils recherchent et ce qui, dans les textes ou l'iconographie, peut faire sens pour eux.

La recherche des traces du passé que les historiens et les archéologues ont en commun - dans le sous-sol pour l'archéologue, dans les textes pour l'historien - et qui est la base de leur travail, poursuit le même objectif : celui de restituer l'histoire. Toutefois dans le détail du quotidien, les deux métiers sont différents. L'archéologue a un savoir-faire bien spécifique : il sait lire un terrain archéologique, interpréter une stratigraphie, faire des liens entre différentes strates. Son travail est par ailleurs très physique et demande une bonne prise en compte de la sécurité et des conditions de travail des personnes. Le responsable d'opération a, en outre, la tâche d'organiser un chantier, une

1. La thèse de Colette Castrucci porte sur les maisons du quartier du port à Marseille à l'époque moderne, quartier qui a été détruit en 1943. Elle a été soutenue en 2010 sous la direction de Régis Bertrand à Aix-Marseille Université. Sa publication en 2016 se trouve sous le titre Le quartier du port de Marseille 1500-1790. Une réalité urbaine restituée, aux Presses Universitaires de Provence, dans la collection Archéologies Méditerranéennes. Le travail s'appuie sur un dialogue entre les documents d'archive (textes réglementaires, cadastre, archives notariées) qui ont permis de restituer, en s'appuyant sur les découvertes de quatre opérations archéologiques, la topographie urbaine, l'organisation des maisons et les techniques de construction mises en œuvre, d'un quartier dont presque aucun élément de bâti ancien ne subsistait. 
équipe, et doit tenir compte d'un certain nombre de paramètres humains, scientifiques et techniques, qu'il doit coordonner.

L'historien est plus solitaire face aux textes des archives. La complexité de sa démarche le conduit à avoir une bonne connaissance des institutions de la période étudiée et de l'organisation des différents fonds d'archives afin de pouvoir naviguer entre eux à la recherche des éléments dont il a besoin.

Son rôle "d'associé" des archéologues est quelquefois ingrat et réducteur puisqu'il doit rechercher en priorité les éléments qui vont faire sens pour les archéologues : plans, cartes, cadastres, plans terriers, etc. Ceux-ci n'ont pas toujours eu conscience que les archives ne sont pas une source infinie d'informations qui va permettre d'apporter des réponses à toutes les questions posées par les vestiges. Plusieurs années d'expérience ont permis toutefois d'établir une meilleure compréhension des choses. En particulier il est devenu évident que l'historien, autant que l'archéologue, est soumis aux aléas de ses découvertes, il ne maîtrise pas à l'avance s'il va trouver dans les archives tous les éléments de compréhension du terrain qu'il souhaiterait. Cette première étape franchie, les archéologues ont compris que l'historien peut aborder une opération archéologique selon un autre angle de vue et élargir ainsi la recherche, en s'intéressant à d'autres aspects qui ne sont pas l'objet stricto sensu de la fouille : histoire du paysage environnant, évolution des parcelles, le pouvoir éminent détenu sur celles-ci, l'évolution urbaine.

\section{RH 19 : Les parcours sont rares qui mêlent histoire et archéologie; com- ment en êtes-vous venue à travailler avec l'INRAP?}

Durant mes études, précisément juste après l'obtention de la licence d'histoire, j'ai eu l'occasion de faire des vacations à la bibliothèque d'histoire, d'archéologie et de lettres classiques du Centre Camille Jullian à Aix-en-Provence où les bibliothécaires recherchaient des vacataires. Cette bibliothèque, gérée conjointement par le CNRS et l'Université de Provence était, ce qui est toujours le cas, fréquentée aussi bien par des étudiants que par des chercheurs. L'une d'entre eux, Antoinette Hesnard ${ }^{2}$, qui m'avait connue dans ce contexte, m'a proposé un jour de travailler avec elle sur un chantier d'archéologie préventive qu'elle allait diriger dans le quartier du port de Marseille. Cette opération a été l'une des premières fouilles d'archéologie préventive à Marseille dans les années 1990. Elle a été codirigée par le CNRS (Antoinette Hesnard) et l'AFAN (Association pour les Fouilles Archéologiques, devenue INRAP en 2002). Là j'ai pu assister Philippe Bernardi qui était chargé de réaliser la recherche en archives $^{3}$. C'est lui qui m’a expliqué la démarche, aidée à mettre au point une méthode et "appris» ce métier qui était en train de se construire. J'ai ensuite été chargée de réaliser l'étude d'archives d'un autre grand chantier marseillais, celui du quartier du port, qui est à l'origine

2. Antoinette Hesnard ( $\dagger$ ) était directrice de recherche au Centre Camille Jullian.

3. Philippe Bernardi, est actuellement directeur de recherche au CNRS (LAMOP - UMR 8589). 
de mon sujet de thèse. La fouille a duré un an et demi. Puis j’ai été engagée à l'AFAN. Ce travail m'a tout de suite à la fois amusée et passionnée car mes découvertes, qui se faisaient en lien avec les vestiges que les archéologues mettaient au jour, pouvaient être associées à la construction d'un puzzle. À chaque découverte, je restituais un petit bout d'histoire du quartier ou d'un bâtiment trouvé en fouilles.

RH 19 : À quelles fouilles concernant au moins partiellement le $\mathrm{XIX}^{\mathrm{e}}$ siècle avez-vous été associée et à quel titre? Ces fouilles sont-elles nombreuses? Est-ce une expérience récente qui change un peu les règles du métier?

J'ai été chargée il y a une dizaine d'années d'effectuer une étude d'archives associée à la fouille d'une raffinerie royale de soufre et de salpêtre fondée sous Louis XIV et ayant fonctionné jusqu'au début du $\mathrm{Xx}^{\mathrm{e}}$ siècle. Un plan de masse daté de 1884 a permis de montrer que les vestiges découverts correspondaient au dernier état de l'usine et d'en détailler l'organisation. De plus, la mise en relation avec les documents issus des archives de la propriété industrielle, et des vestiges ont fourni des renseignements essentiels sur les méthodes de raffinage utilisées durant le XIX ${ }^{\mathrm{e}}$ siècle. Cette fouille a été d'autant plus exceptionnelle que l'établissement industriel n'avait pas été repéré lors du diagnostic préalable à la fouille. L'objectif de celle-ci était de mettre au jour un site du Néolithique! Le temps prévu pour l'opération a été augmenté afin de fouiller les vestiges modernes qui affleuraient de manière spectaculaire. C'était la première fois qu'une fouille de ce type se déroulait à Marseille et qu'une telle recherche, associant étude des sources écrites et archéologie du XIX $x^{e}$ siècle était pratiquée. Nous y avons été aidés par l'un des spécialistes du patrimoine industriel méditerranéen, Xavier Daumalin ${ }^{4}$, venu reconnaître le site dès les premiers jours, et par Patrice Bret, qui nous a permis d'accéder à un plan de l'usine conservé au Comité Lavoisier ${ }^{5}$, plan qui a été essentiel dans la compréhension du site. Mis en perspective avec les vestiges, celui-ci s'est révélé être le plan de la manufacture le plus récent établi avant sa destruction (fig. 1).

J'ai également été chargée de l'étude d'archives associée au diagnostic réalisé sur l'une des dernières savonneries marseillaises, située dans le cœur de ce qui a été au XIX siècle, le quartier des savonneries. Un plan de Demarets datant de 1808, ainsi que les planches du dictionnaire d'Alembert et Saglio, ont permis de restituer l'état probablement le plus récent de la savonnerie et d'identifier les structures découvertes dans la chaîne de fabrication du savon : les «mises", structures permettant de faire prendre le savon, et les chaudières,

4. Xavier Daumalin est professeur d'histoire contemporaine à Aix-Marseille Université, directeur de recherche et directeur de l'UMR 7303 et secrétaire général du Comité Lavoisier.

5. Patrice Bret est responsable scientifique du département d'histoire du Centre des hautes études de l'armement. 


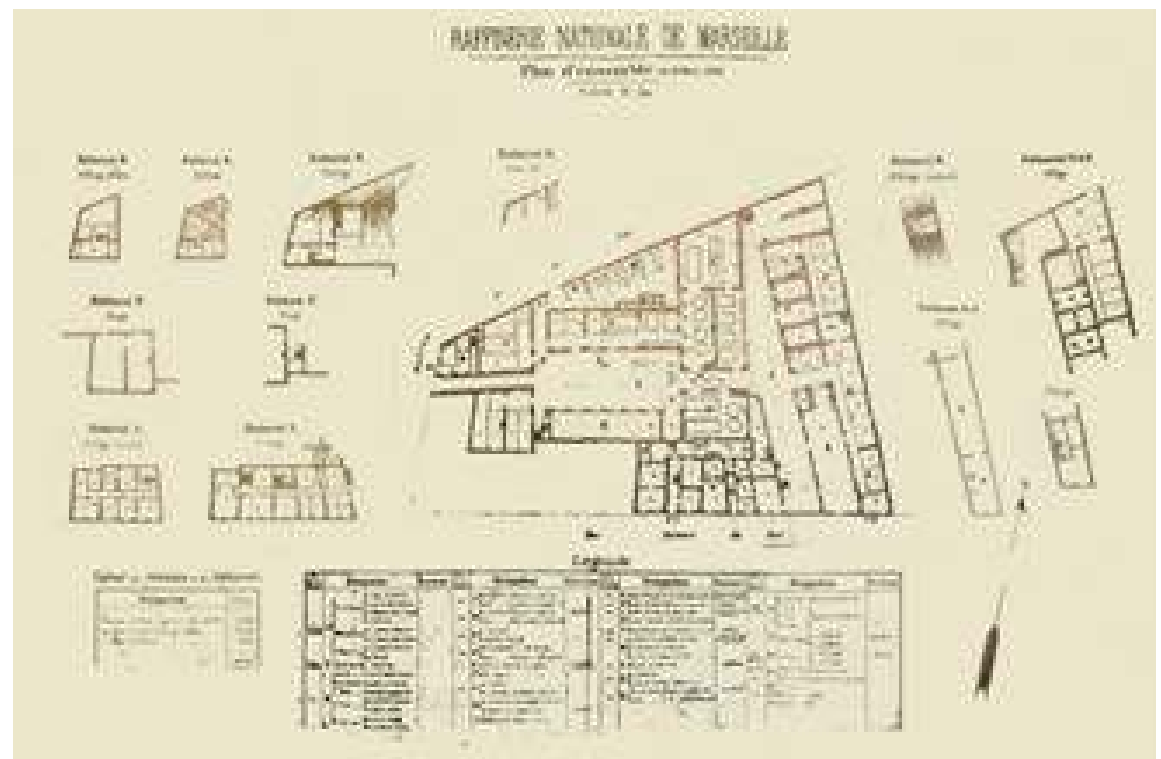

Fig. 1 : Plan de la Raffinerie de salpêtre et de soufre de Marseille, 1884 (Département d'histoire de I'Armement)

cuves de 3 mètres de diamètre, associées à un réseau de galeries souterraines (fig. 2).

Quant au cimetière des Petites Crottes situé dans un des quartiers au nord de Marseille, qui a fonctionné de la fin du XviII au début du Xxe siècle, ce sont des plans établis au XIX ${ }^{e}$ siècle à l'occasion des divers projets d'agrandissement du cimetière, et conservés aux Archives municipales de Marseille, qui ont permis, en les associant aux états de section du cadastre napoléonien ${ }^{6}$, d'en appréhender l'évolution.

La fouille de la manufacture en 2007 a marqué un tournant dans la prise de conscience par les autorités scientifiques et par l'État de l'intérêt de fouiller ce type de structures. La nécessité de rechercher dans les archives des documents iconographiques ou textuels susceptibles d'expliquer le contexte historique de leur installation et leur fonctionnement est apparu évidente. L'importance des découvertes - pérennité d'une manufacture créée à la fin du $\mathrm{XVIII}^{\mathrm{e}}$ siècle et fermée au début du $\mathrm{XX}^{\mathrm{e}}$ siècle, organisation de l'usine, procédés industriels utilisés, implication dans le système de fabrication de la poudre de guerre - a mis en évidence l'utilité de se réapproprier ce passé proche dont la culture matérielle était déjà en grande partie oubliée. Les sources d'archives sollicitées, issues des Archives municipales de Marseille et du Service Historique de la Défense, ont conduit à attester l'activité de l'usine à partir des

6. Archives Municipales de Marseille (AMM), 52 M 647; 8 D 1, 16, 2; 1 D 96.

7. AMM, EE 83. 


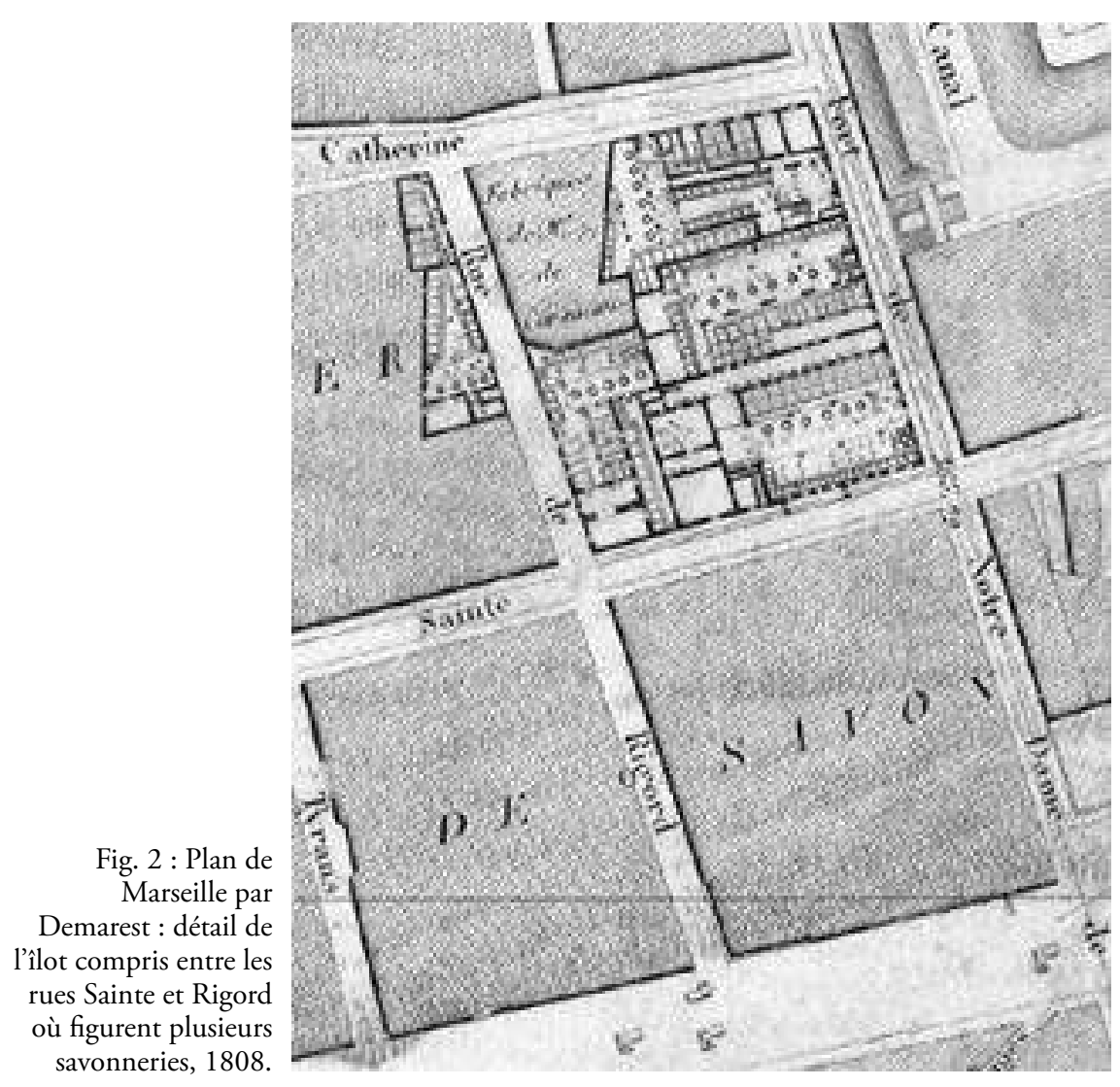

années 1690, jusqu'au début du Xxe siècle. Manufacture royale d'abord, créée sous Louis $\mathrm{XIV}^{8}$, elle a traversé les âges en demeurant un organisme d'État en relation avec la Poudrerie de Saint-Chamas à qui elle a longtemps livré ses produits raffinés nécessaires à la fabrication de la poudre de guerre.

L'apport du plan de masse de 1884, conservé au Département d'histoire de l'Armement (voir fig. 1), a été déterminant. Très bien légendé, il a permis la restitution de l'organisation interne de l'usine. On a pu constater que les vestiges mis au jour correspondaient exactement à ce plan qui a dû être le dernier réalisé avant sa fermeture.

La figure 3 renvoie à l'ensemble de la fouille. On y voit un carneau central, par où sont évacuées les fumées dirigées vers une grande cheminée, qui divise l'espace en deux parties distinctes : en haut de l'image, la base, la partie réservée au raffinage du salpêtre avec les ouvertures circulaires correspondant aux conduits des fourneaux; la partie réservée au raffinage du soufre se trouve 


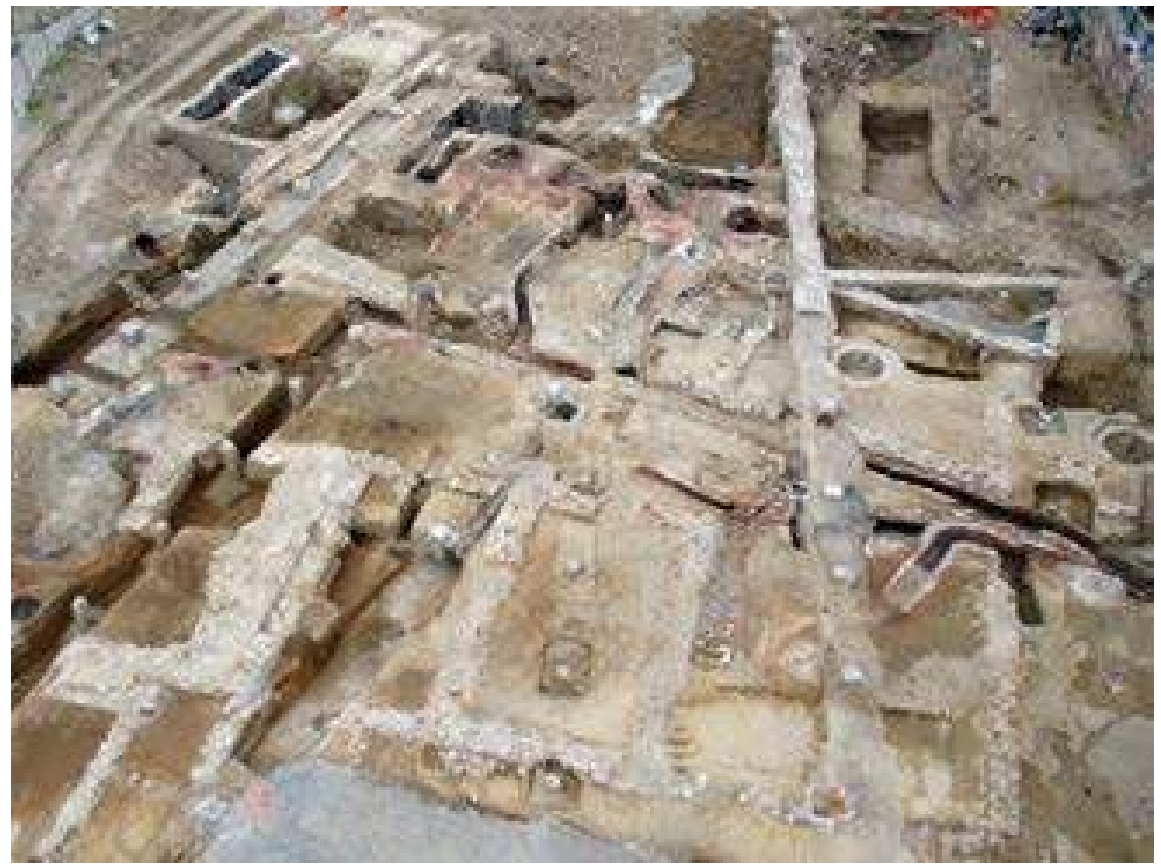

Fig. 3 : Plan de l'ensemble de la fouille de la raffinerie de salpêtre et de soufre de Marseille (cl. F. Parent, Inrap).

au bas de l'image avec les bassins rectangulaires (fig. 3). Le plan de masse représenté sur la figure 4 restitue ces deux parties (fig. 4).

Quant aux Archives nationales de la propriété industrielle (INPI), elles ont livré les brevets déposés respectivement par Michel et Desjardin pour le raffinage du soufre et du salpêtre au XIX $x^{e}$ siècle. À la lecture conjointe du plan de 1884, de celle de deux rapports de visite de la raffinerie par des commissaires aux poudres à cinquante ans d'intervalle au cours du XIX $\mathrm{X}^{\mathrm{e}}$ siècle, on a pu vérifier que les deux procédés avaient été employés successivement dans cet établissement ${ }^{9}$.

Depuis cette fouille, des diagnostics sont plus souvent prescrits sur des terrains où la présence de sites industriels ou d'habitations datant du XIx siècle est connue. Le zonage archéologique a été étendu, notamment à Marseille et les zones périurbaines occupées jusqu'au milieu du XIX siècle par de grandes propriétés agricoles et par des bâtiments - bastides ou bâtiments agricoles sont maintenant de plus en plus fréquemment concernées. L'essor des villes, au milieu du XIX ${ }^{\mathrm{e}}$ siècle, transforme ce terroir en quartier industriel ou au contraire en secteur résidentiel où se produisent des événements spécifiques :

9. Service Historique de la Défense, 4 W 580. La fouille de la manufacture a fait l'objet d'une publication : Ingrid SENEPART (dir.), La raffinerie de soufre et de salpêtre de Marseille, Aix-enProvence, BIAMA, 2016. 


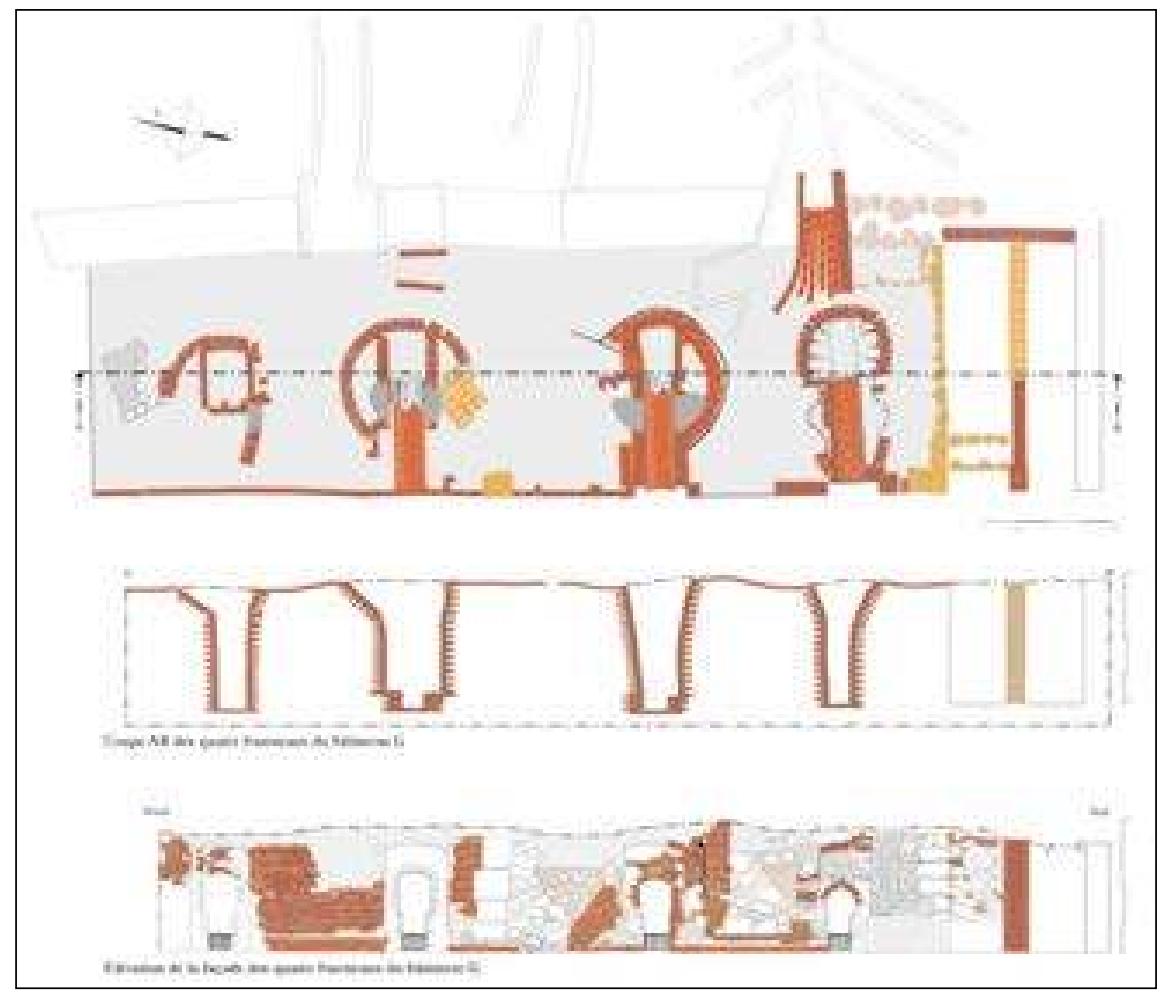

Fig. 4 : Plan de masse des vestiges de la raffinerie au XvıII ${ }^{\mathrm{e}}$ siècle (relevé et D.A.O L. Vallieres, Inrap).

construction d'usines, logements, création de cimetières à distance des habitations, ou encore de résidences secondaires. La recherche dans les cadastres ou les délibérations municipales par exemple, permet d'être au plus près de l'évolution de ces secteurs et de saisir des éléments qui ne sont plus visibles sur le terrain. Ainsi cette carrière de travertin découverte dans le quartier de Saint Barnabé/Saint Julien situé à l'Est de Marseille, extra-muros, qui était un secteur rural jusqu'au début du $\mathrm{xx}^{\mathrm{e}}$ siècle. On l'imaginait antique. En fait un décret du 17 août 1864, enjoignant les propriétaires d'une carrière d'en faire la déclaration, montre qu'elle avait appartenu au milieu du XIx ${ }^{e}$ siècle à un particulier qui l'avait fait ouvrir lors de la construction de sa maison secondaire, puis exploitée pour en tirer profit, le secteur étant alors en pleine expansion immobilière ${ }^{10}$, mais sa durée de vie avait été suffisamment courte pour échapper à tout recensement cadastral (fig. 2).

Le nombre des opérations archéologiques concernant le $\mathrm{XIX}^{\mathrm{e}}$ siècle a donc augmenté sans pour autant modifier les méthodes utilisées. 
RH 19 : Vous avez participé notamment à une archéologie attachée à l'architecture. Qu'est-ce qu'une archéologie du hors-sol? Que fouille$t$-on et que cherche-t-on quand on fouille de l'architecture, qu'il s'agisse de sites industriels comme de quartiers d'habitation?

En l'occurrence, les fouilles attachées à l'architecture auxquelles j'ai été associée recélaient des vestiges uniquement souterrains. Il s'agissait d'un secteur situé autour de l'Hôtel de ville de Marseille qui a été, à quelques exceptions près, entièrement détruit en 1943. Seuls quelques édifices en place dont l'Hôtel de ville, et quelques hôtels particuliers dont l'Hôtel de Cabre et la Maison dite Diamantée avaient subsisté. Les fouilles ont mis au jour essentiellement des caves, des fondations, des traces de la voierie. La restitution d'une grande partie des maisons ainsi que l'organisation urbaine de ce secteur a pu se faire grâce à des registres de cens et des documents réglementaires concernant les aménagements de la voierie. Mais ce sont surtout les archives notariées qui ont apporté le plus d'éléments permettant de restituer les élévations : prix-faits, rapport de future cautelle (documents analogues aux rapports d'estime de la Chambre des bâtiments pour Paris, mais ici passés devant notaire). Une restitution de l'ensemble du quartier le plus convoité de Marseille durant l'Ancien Régime a pu être réalisée. Les informations fournies par les textes notariés ont renseigné aussi bien l'organisation de la maison que les techniques de construction, des fondations jusqu'au toit. Plus d'une centaine d'habitations privées a été suivie sur une période allant de la fin du XVI ${ }^{\mathrm{e}}$ siècle au milieu du XVIII ${ }^{\mathrm{e}}$ siècle. L'histoire des bâtiments a été retracée, mais aussi l'évolution des manières d'habiter et celles de construire.

Quant à la question concernant la fouille d'un bâtiment en élévation, donc une "étude de bâti", il s'agit de rechercher les signes d'une rupture dans le bâti qui correspondrait à une transformation de l'édifice. En décroutant les différents enduits, les archéologues essaient de repérer une modification dans la technique de construction. Dans le même objectif, ils fouillent également le sous-sol à la recherche de sépultures par exemple, ou d'installations industrielles plus anciennes ou d'un autre type que celui le plus visible. Les archives cadastrales, les plans terriers, peuvent fournir des éléments permettant une généalogie du bâtiment. Pour l'Ancien régime les établissements religieux ou hospitaliers tenaient des registres de cens dans lesquels sont consignés les différents emphytéotes des biens sur lesquels ils ont le pouvoir éminent. Il en est de même pour les propriétés relevant du pouvoir direct du roi.

Pour les établissements industriels du XIx e siècle, il n'y a pas eu obligation donnée aux entreprises de verser leurs archives. On peut trouver des informations concernant les circonstances de leur installation, l'histoire de leur construction ou des plans d'aménagements, dans les fonds des établissements classés insalubres, où se trouvent également les autorisations d'installer une usine polluante. 
Fig. 5 : Vue du sudouest du sous-sol du bâtiment $\mathrm{G}$ avec la façade des fourneaux (cliché Fr. Parent, Inrap)

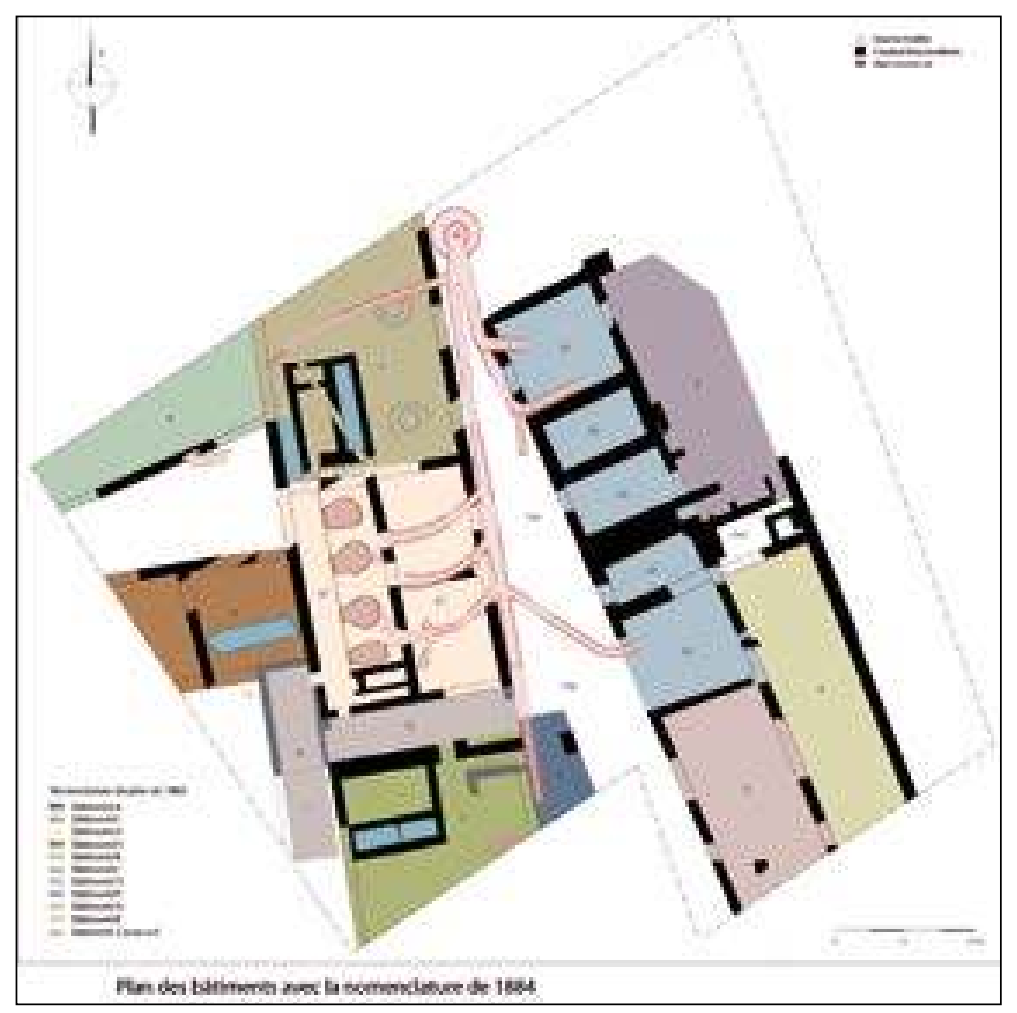

Par ailleurs, le Service historique de la Défense conserve les archives des établissements liés à l'armement, à la défense, comme cela a été le cas pour la Manufacture de soufre et de salpêtre, de même que des documents concernant les fortifications.

La situation est différente à chaque fois. On peut dire que c'est en fonction des archives disponibles que le travail s'organise. Encore une fois, il est évident que les archives ne sont pas exhaustives, on ne sait pas d'avance ce que l'on va trouver.

En général on recherche comment le, ou les, bâtiments sont organisés. Soit par des rapports d'experts, soit par des plans dressés lors de la création ou de l'agrandissement des bâtiments industriels. J'ai souligné le rôle joué par le plan de 1884 dans la lecture de la raffinerie de soufre. En le comparant au plan des vestiges, chaque espace de travail a pu être replacé, on a pu remarquer deux secteurs distincts, celui pour le raffinage du salpêtre et celui pour le raffinage du soufre. Ainsi les installations trouvées dans les sous-sols ont pu être reliées à celles qui n'existaient plus en élévation mais dont on avait des représentations dans les archives industrielles et dans les dictionnaires spécialisés. La figure 5 représente la restitution de l'entrée des fourneaux et de leurs conduits en sous-sol (fig. 5). 
Parallèlement, un autre aspect de l'étude sur l'architecture vise à repérer les techniques et matériaux de construction, qu'il s'agisse d'une zone importante comportant plusieurs bâtiments ou qu'il ne s'agisse que d'un bâtiment industriel. L'objectif étant de déterminer à quel moment une technique a évolué.

RH 19 : Comment avez-vous travaillé et travaillez-vous encore (sur le terrain ou en archives)? Y a-t-il une procédure ou un cadre spécifique contraignant?

Comme je l'ai dit précédemment, à chaque fouille correspond une étude d'archives différente. J'adapte ma méthode de travail à chaque cas. Car l'approche ne se fait pas seulement en fonction de ce que l'on cherche, mais également selon les sources disponibles. Cette remarque est valable pour toutes les périodes y compris pour l'Ancien Régime. Les archives du XIX $X^{\mathrm{e}}$ siècle sont comprises dans les fonds regroupant tous les documents postérieurs à 1790 et allant jusqu'à 1940 .

Lorsqu'il n'y a pas de sources directement attachées à un lieu, un bâtiment, une institution, etc., il faut aborder l'étude par un autre angle : délibérations municipales, cadastres, textes concernant la voirie, autorisations d'installer un établissement polluant, etc., qui contiennent des dossiers quelquefois très complets avec des plans et des détails sur les propriétés voisines. Mais j'insiste sur le fait qu'il n’y a pas de difficultés particulières liées au XIX ${ }^{e}$ siècle.

RH 19 : Comment se passe le dialogue avec les archéomètres et les archéologues, à la fois sur le plan intellectuel et, plus concrètement, dans le quotidien des chantiers?

Le dialogue se fait dans la confiance car mon expérience à l'INRAP m'a fait travailler avec plusieurs archéologues qui connaissent bien maintenant les difficultés et les aléas de la recherche en archives, tout en étant conscients de la richesse des informations qu'elle offre. Donc, en général, le dialogue est très souple. Je rencontre le responsable de l'opération archéologique en amont. Il me présente le site à étudier, avec les premiers éléments dont il dispose : s'il s'agit d'une fouille, il m'exposera les résultats du diagnostic qui a précédé et me fera part de ses attentes. S'il s'agit d'un diagnostic, le contenu de la prescription faite par le conservateur servira de base à ma recherche. Le problème le plus important dans le cadre de l'archéologie préventive est celui du temps. Le temps consacré aux opérations archéologiques et aux études associées est de plus en plus réduit. Je me trouve souvent face à une demande dont je sais d'avance qu'elle ne pourra pas être satisfaite par manque de temps. Je fais un premier repérage dans les archives, puis je vois avec le responsable d'opération le sens dans lequel peut aller la recherche, les limites qu'elle va avoir, etc. Quand il y a le choix entre deux ou trois directions possibles et que le temps à consacrer à l'étude est limité, il m’indique quelles sont ses priorités. 
De manière générale, que ce soit durant la fouille ou en post-fouille pour la phase d'analyse, le dialogue reste ouvert entre les archéologues et moimême afin de tenter de répondre à la fois aux questions qu'ils posent mais également de décider de thèmes de recherche à développer lors de la synthèse générale en vue d'une éventuelle publication. Lorsqu'elle est possible, c'est le cas lorsqu'il s'agit d'une fouille de grande ampleur, c'est l'occasion d'approfondir certains points.

RH 19 : D'après votre expérience, quelle est la place du XIX ${ }^{e}$ siècle dans les chantiers auxquels vous avez été associée? (Un passage obligé? Une portion congrue? L'objet du chantier?)

Jusqu'à il y a une dizaine d'années, le XIX siècle n'était pas, en tout cas dans le cadre de l'archéologie préventive, retenu comme étant une période à étudier. Il n'y avait donc pas d'opérations archéologiques qui lui étaient consacrées. Toutefois, pour comprendre l'enchevêtrement de la stratigraphie, on cherchait dans les archives des traces des bâtiments les plus récents, dont les élévations n'existaient parfois plus mais dont la construction avait laissé des traces dans le sous-sol. L'intérêt pour le XIx ${ }^{e}$ siècle est relativement récent chez les archéologues, à Marseille en particulier et a été provoqué par la découverte de la raffinerie de salpêtre et de soufre qui a eu vraiment un effet déclencheur. Toutefois cet intérêt est réel. Depuis la découverte de ce site exceptionnel, il y a eu une prise de conscience de la part des archéologues. La redécouverte d'éléments d'un passé très proche construit un lien entre le passé beaucoup plus éloigné duquel ils étaient plus coutumiers et l'époque actuelle. C'est en quelque sorte le maillon manquant qui permet de mieux comprendre comment s'articulent les mutations urbaines et rurales. Aussi le $\mathrm{XIX}^{\mathrm{e}}$ siècle, de même que le $\mathrm{XX}^{\mathrm{e}}$, sont devenus des objets archéologiques. Et des opérations archéologiques, diagnostic ou fouille, accompagnées a minima d'une étude documentaire, si ce n'est d'une étude d'archives, sont plus systématiquement décidées dès qu'il y a connaissance, ou même suspicion de l'existence de structures industrielles ou militaires récentes sur un terrain où une construction est projetée. Il faut souligner toutefois que cela dépend beaucoup du prescripteur.

RH 19 : Quels sont les problèmes spécifiques que pose la fouille du $\mathrm{XIX}^{\mathrm{e}}$ siècle?

La méthode de travail est la même et ne requiert aucune technique particulière. L'enregistrement des vestiges se fait de la même manière, selon les mêmes méthodes. Pour ce qui concerne les archives, comme je l'ai dit, elles sont classées dans les fonds et séries contenant les documents datant d'après la période révolutionnaire. Elles présentent l'avantage d'être en langue française moderne; il n'y a pas la barrière de la connaissance des écritures anciennes à franchir. 
Toutefois, il faut prendre en compte le fait que les innovations du $\mathrm{XIX}^{\mathrm{e}}$ siècle, comme pour toutes les périodes, prennent quelquefois leur source dans la période antérieure, ou sont le résultat de l'évolution d'une technique mise au point antérieurement. Il faut donc aller chercher plus en amont.

Il y a par ailleurs et avant tout à sensibiliser les services de l'État sur l'intérêt de prescrire des opérations archéologiques lors de travaux d'aménagement des zones périurbaines; l'archéologie a un retard à rattraper concernant la prise en compte de la culture matérielle et industrielle du XIx ${ }^{\mathrm{e}}$ siècle par rapport aux études historiques qui s'en sont emparés depuis plusieurs décennies montrant tout l'intérêt de se réapproprier ce passé très proche. 